\title{
APPLICATION OF GPS SENSOR FOR RECOVERY OF PRECISE POSITION OF AIRCRAFT IN AIR TRANSPORT
}

\author{
Kamil Krasuski ${ }^{1}$, Janusz Cwiklak ${ }^{2}$ \\ ${ }^{1}$ District Office in Ryki, Poland; ${ }^{2}$ Polish Air Force Academy, Poland \\ kk_deblin@wp.pl,j.cwiklak@wsosp.pl
}

\begin{abstract}
The GNSS satellite technique is applied for a designation of the true position of the aircraft vehicle in air transport. In case of air transport, the GNSS satellite technique is implemented within the ABAS, SBAS and GBAS augmentation systems. The aim of this paper is to present and describe the results of applying the GNSS satellite technique in the ABAS augmentation system in air transport. The research experiment was conducted on 1st June 2010 (01.06.2010) at late morning hours on the airfield in the town of Dęblin in the Lubelskie Voivodeship, in south-eastern Poland. Within the research experiment, nearby Dęblin airfield a flight test was executed using a Cessna 172 plane. During the flight test, the dual frequency Topcon HiperPro receiver was installed in the pilot's cabin of the Cessna 172 aircraft. The Topcon HiperPro receiver collected a GPS code, phase and Doppler measurements in order to recover the true position of the aircraft. In this paper the authors present the results of positioning the Cessna 172 during the phase of landing based on the solution of the GPS satellite technique. The position of the aircraft was estimated by means of the Precise Point Positioning (PPP) method in GAPS software for un-difference GPS code and phase observations. The PPP method is applied in air navigation as a precise technique for recovery of position of aircrafts. Especially, designation of true position of the aircraft is very important at the phase of landing on the aerodrome. In this paper, the obtained accuracy for $X Y Z$ geocentric coordinates of the aircraft is better than $6 \mathrm{~cm}$ at the phase of landing. The precision of the aircraft coordinates from GAPS software was compared with the values from magicPPP and CSRS-PPP softwares. The dispersion of the difference of the aircraft coordinates between GAPS and CSRS-PPP softwares is between -0.5 $\mathrm{m}$ and $+1.2 \mathrm{~m}$. In addition, the dispersion of the difference of the aircraft coordinates between GAPS and magicPPP softwares is between $-0.9 \mathrm{~m}$ and $+1.8 \mathrm{~m}$.
\end{abstract}

Keywords: GPS, air transport, PPP method, accuracy.

\section{Introduction}

The use of navigation systems in air transport would not be possible without an introduction of auxiliary augmentation modules supporting aircraft positioning. The examples of support systems used in air transport are ABAS (Aircraft-Based Augmentation System), SBAS (Satellite-Based Augmentation System) and GBAS (Ground-Based Augmentation System) systems [1;2]. The ABAS system is particularly favoured for its application in aircraft on-board avionics, being additionally coupled with the GNSS receiver or the INS (Inertial Navigation System) sensor. The SBAS ensures the application of differential correction data from geostationary satellites, e.g., EGNOS (European Geostationary Navigation Overlay Service) or WAAS (Wide Area Augmentation System), to determine the aircraft position during a flight. On the other hand, the GBAS system allows determining the aircraft coordinates on the basis of differential corrections from the physical reference station network, e.g., the ASG-EUPOS (Aktywna Sieć Geodezyjna - EUPOS) system [3].

One of the new application solutions used in the augmentation system ABAS is the Precise Point Positioning (PPP) method. The PPP method provides high accuracy in calculating the coordinates of the aircraft [4]. Moreover, the PPP method is excellent in specifying the atmosphere parameters as well as making corrections in the receiver clock bias. The PPP method is exploited in a number of scientific fields, such as: geophysics, meteorology, precise time transfer, navigation, climatology, geodesy, cartography, etc [5].

This article is intended to discuss and describe the method of Precise Positioning as a sample use of the ABAS system in air transport. Furthermore, we presented the results of the positioning of the Cessna 172 aircraft during an approach to landing. In the experiment we used dual-frequency codephase GPS observations of the GNSS receiver in order to recover a credible position of the Cessna 172 aircraft. The flight test was carried out at the military airfield in Dęblin in 2010.

\section{Materials and methods}

A mathematical model of determining the aircraft position is based on the linear Ionosphere-Free combination for un-difference code-phase observations in the GPS (Global Positioning System) navigation system as follows [6]: 


$$
\left\{\begin{array}{c}
P_{3}=\alpha \cdot P 1+\beta \cdot P 2=\rho+c \cdot(d t r-d t s)+\operatorname{Trop}+\operatorname{Re} l+M_{P 3} \\
L_{3}=\alpha \cdot L 1+\beta \cdot L 2=\rho+c \cdot(d t r-d t s)+\operatorname{Trop}+\operatorname{Re} l+B_{3}+\delta_{w u}+M_{L 3}
\end{array},\right.
$$

where $P_{3}$ - Ionosphere-Free linear combination for code observations, unit, $\mathrm{m}$;

$L_{3}$ - Ionosphere-Free linear combination for phase observations, unit, m;

$\left(P_{1}, P_{2}\right)$ - code observations at $1^{\text {st }}$ and $2^{\text {nd }}$ frequency in GPS system, unit, $\mathrm{m}$;

$\left(L_{1}, L_{2}\right)-$ phase observations at $1^{\text {st }}$ and $2^{\text {nd }}$ frequency in GPS system, unit, $m$;

$(\alpha, \beta)$ - coefficients of linear combination, unit;

$\rho$ - geometric distance between the satellite and receiver, unit, $\mathrm{m}$;

$\rho=\left(\left(x-X_{G P S}\right)^{2}+\left(y-Y_{G P S}\right)^{2}+\left(z-Z_{G P S}\right)^{2}\right)^{0.5}$, unit, $\mathrm{m}$;

$(x, y, z)$ - aircraft vehicle position referenced to geocentric coordinates, unit, $\mathrm{m}$;

$\left(X_{G P S}, Y_{G P S}, Z_{G P S}\right)$ - satellite coordinates in GPS system, unit, $\mathrm{m}$;

$c$ - speed of light, unit, $\mathrm{m} \cdot \mathrm{s}^{-1}$;

$d t r$ - receiver clock bias, unit, s;

$d t s$ - satellite clock bias, unit, s;

$B_{3}$ - float ambiguity, unit, m;

Trop - distance correction to compensate delay due to troposphere effects, unit, m;

Rel - relativistic effect, unit, $\mathrm{m}$;

$\delta_{w u}$ - phase wind up, unit, m;

$M_{P 3}-$ multipath effect for code observations, unit, $\mathrm{m}$

$M_{L 3}$ - multipath effect for phase observations, unit, $\mathrm{m}$.

The use of precision products of IGS (International GNSS Service) services, i.e. precise ephemeris and clock parameters, in the Ionosphere-Free linear combination considerably increases the accuracy of computing the final products from the equation (1). Application of precise IGS services products in the Ionosphere-Free linear combination for undifferentiated GNSS (Global Navigation Satellite System) observations is named the method of Precise Point Positioning (PPP) [7]. The PPP method is increasingly used in satellite measurements using the GNSS observation only from one receiver. Besides, it needs to be stressed that the PPP method provides high accuracy of determining the user position in the static mode and kinematic mode.

The total number of unknown parameters in equation (1) equals:

- 3 parameters of the aircraft coordinates in ECEF (Earth-Centered-Earth-Fixed) geocentric frame,

- 1 parameter of receiver clock bias,

- 1 parameter of ZWD (Zenith Wet Delay) as a product of troposphere delay,

- ambiguity term is estimated for each satellite in each measurement epoch.

The mathematical model of determining the required parameters from the equation (1) is based on the method of least squares in the sequential process for all measurement epochs, as below [8]:

$$
\boldsymbol{\delta} \mathbf{x}=\left(\mathbf{A}^{T} \cdot \mathbf{P} \cdot \mathbf{A}+\mathbf{C}_{x, t}^{-1}\right)^{-1} \mathbf{A}^{T} \cdot \mathbf{P} \cdot \mathbf{l},
$$

where $\mathbf{\delta} \mathbf{x}-$ vector with unknown parameters;

A - matrix of coefficients;

$\mathbf{P}$ - matrix of weights;

$\mathbf{C}_{x, t}$ - covariance matrix of unknown parameters at current measurement epoch;

$t$ - current measurement epoch;

$\mathbf{C}_{x, t}=\left(\mathbf{A}^{T} \cdot \mathbf{P} \cdot \mathbf{A}+\mathbf{C}_{x,(t-1)}^{-1}\right)^{-1}+\mathbf{C}_{n} ;$

$\mathbf{C}_{x,(t-1)}$ - covariance matrix of unknown parameters from previous measurement epoch;

$(t-1)$ - previous measurement epoch;

$\mathbf{C}_{n}$ - matrix of process noise;

I - misclosure vector. 
In turn, the accuracy of the computed coordinates of the aircraft is solved from equation (2), in the following way:

$$
m x=\sqrt{\mathbf{C}_{x, t}(1,1)} ; \quad m y=\sqrt{\mathbf{C}_{x, t}(2,2)} ; \quad m z=\sqrt{\mathbf{C}_{x, t}(3,3)},
$$

where $m x-$ standard deviation along to $X$ axis;

$m y$ - standard deviation along to $Y$ axis;

$m z$ - standard deviation along to $Z$ axis.

It should be noted that the values of the parameters $(m x, m y, m z)$ are expressed using orthocartesian coordinates $X Y Z$ in relation to the ECEF geocentric system.

Within the research experiment we determined the coordinates of the aircraft in the PPP method. For this purpose, we used undifferentiated GPS observations made from a dual-frequency Topcon HiperPro receiver fixed on board the Cessna 172 aircraft (see Fig. 1).

\section{Antenna and Topcon receiver}

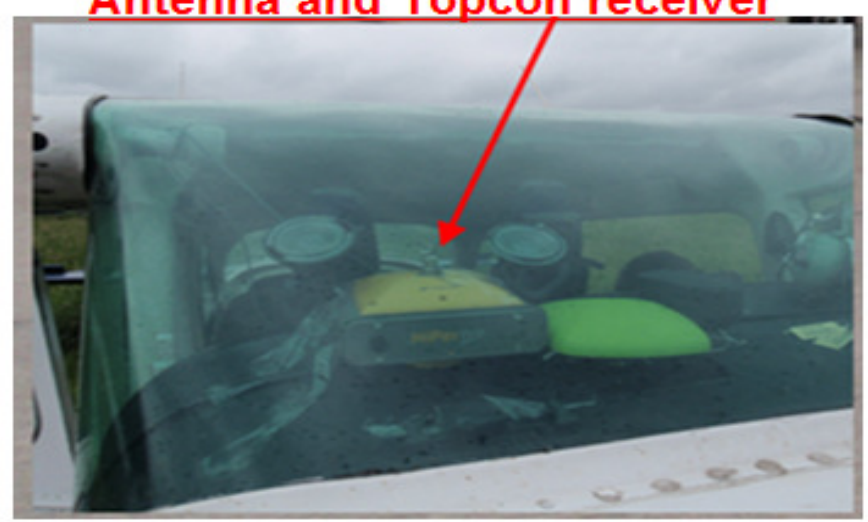

Fig. 1. Antenna and Topcon receiver in pilot's cabin in Cessna 172 plane

The test flight was made on June 1 2010, near the town of Dęblin [9]. The Topcon HiperPro receiver recorded GPS observations in order to recover the reliable and true position of the aircraft. The registration of GPS observations was made at 1 second intervals for code, phase and Doppler measurements.

The code-phase observations stored in the RINEX (Receiver Independent Exchange System) file were used to determine the position of the aircraft in the GAPS (GNSS Analysis and Positioning Software) v6.0.0 programme. The GAPS programme was developed by researchers from the University of New Brunswick in Canada. Currently the GAPS programme is a free computational service for potential users, available on the website: http://gaps.gge.unb.ca/submitbasic.php. The GAPS program exploits numerical computational strategies as well as configuration for the input parameters [10], as follows:

- GNSS system: GPS system,

- file of RINEX type: RINEX 2.11,

- positioning mode: kinematic,

- computation mode: post-processing,

- source of precise orbits and clocks: GPS precise orbits and clocks as a IGS Final product,

- interval of precise orbits and clocks product: 15 minutes,

- methods of estimation GPS satellites orbits and clocks: Lagrange polynomial method,

- GPS observations: code (P1/P2) and phase (L1/L2) observations,

- linear combination: Ionosphere-Free,

- adjustment processing: applied,

- a priori standard deviation of code measurement: $2 \mathrm{~m}$,

- a priori standard deviation of phase measurement: $1.5 \mathrm{~cm}$,

- cut off elevation: 100 ,

- interval of computation: $1 \mathrm{~s}$,

- maximum number of iterations: 5 , 
- phase center offset/variation for satellites and receiver: based on IGS ANTEX (Antenna Exchange Format) file,

- initial position of aircraft: based on header of RINEX file,

- reference frame: IGS08,

- mathematical model of computations: least square estimation in sequential processing,

- receiver clock bias: estimated,

- ambiguity term: float number,

- output coordinates of aircraft: geocentric $(X Y Z)$ and geodetic (BLh) coordinates,

- reference time: GPS Time,

- instrumental biases DCB (Differential Code Biases): eliminated in Ionosphere-Free linear combination,

- ionosphere delay: 1st term of total ionosphere delay is eliminated in Ionosphere-Free linear combination,

- higher order ionosphere effects: not applied,

- phase wind up: applied,

- relativistic effect: applied,

- ocean and body tide loading: not applied,

- Sagnac effect: applied,

- troposphere model: VMF1-gridded - VMFG_20100601,

- a priori value of troposphere delay: $2.332 \mathrm{~m}$,

- a priori of standard deviation of troposphere delay: $0.1 \mathrm{~m}$,

- mapping function: Vienna Mapping Function,

- troposphere gradients: not applied,

- process noise of Zenith Wet Delay product: $5.0 \mathrm{~mm} / \mathrm{sqrt}(3600 \mathrm{~s})$.

\section{Results and discussion}

Within the conducted experiment we determined the accuracy and precision of computing the coordinates of the aircraft Cessna 172 during an approach to landing. The vertical trajectory of Cessna 172 plane at the landing phase is presented into Figure 2. Determining a reliable position of the aircraft in the approach to the landing phase has a tremendous impact on the safety of air operations. The obtained research findings with regard to the aircraft positioning with the GPS satellite technique are therefore relevant from the practical viewpoint for the ABAS augmentation system in air transport.

Figure 3 shows the values of the dilution of precision DOP (Dilution of Precision) coefficients on the basis of the solution in the GAPS programme. For this purpose, we present the results for the dilution coefficients GDOP, HDOP, VDOP as well as PDOP [11]. The values of the DOP coefficients are determined from the dependence:

$$
\left\{\begin{array}{c}
G D O P=\sqrt{\mathbf{G}(1,1)+\mathbf{G}(2,2)+\mathbf{G}(3,3)+\mathbf{G}(4,4)} \\
P D O P=\sqrt{\mathbf{G}(1,1)+\mathbf{G}(2,2)+\mathbf{G}(3,3)} \\
H D O P=\sqrt{\mathbf{G}(1,1)+\mathbf{G}(2,2)} \\
V D O P=\sqrt{\mathbf{G}(3,3)}
\end{array},\right.
$$

where $G D O P-$ Geometric Dilution of Precision;

$P D O P$ - Position Dilution of Precision;

$H D O P$ - Horizontal Dilution of Precision;

$V D O P$ - Vertical Dilution of Precision;

$\boldsymbol{G}=\left(\boldsymbol{A}^{T} \cdot \boldsymbol{A}\right)^{-1}-$ full rank matrix.

The mean value of the GDOP coefficient is 2.9 , with the dispersion of the obtained results ranging from 2.6 to 3.1. In addition, the median for the GDOP value parameter is equal to 3.0. The mean value of the HDOP coefficient is 2.0, with the dispersion of the obtained results ranging from 2.0 to 2.1. In addition, the median for the HDOP value parameter is equal to 2.0. The mean value of the VDOP coefficient is 1.4 , with the dispersion of the obtained results ranging from 1.1 to 1.7. Moreover, the 
median for the VDOP value parameter equals 1.6. The mean value of the PDOP coefficient is 2.5, with the dispersion of the obtained results ranging from 2.3 to 2.7. Moreover, the median for the PDOP value parameter equals 2.6.

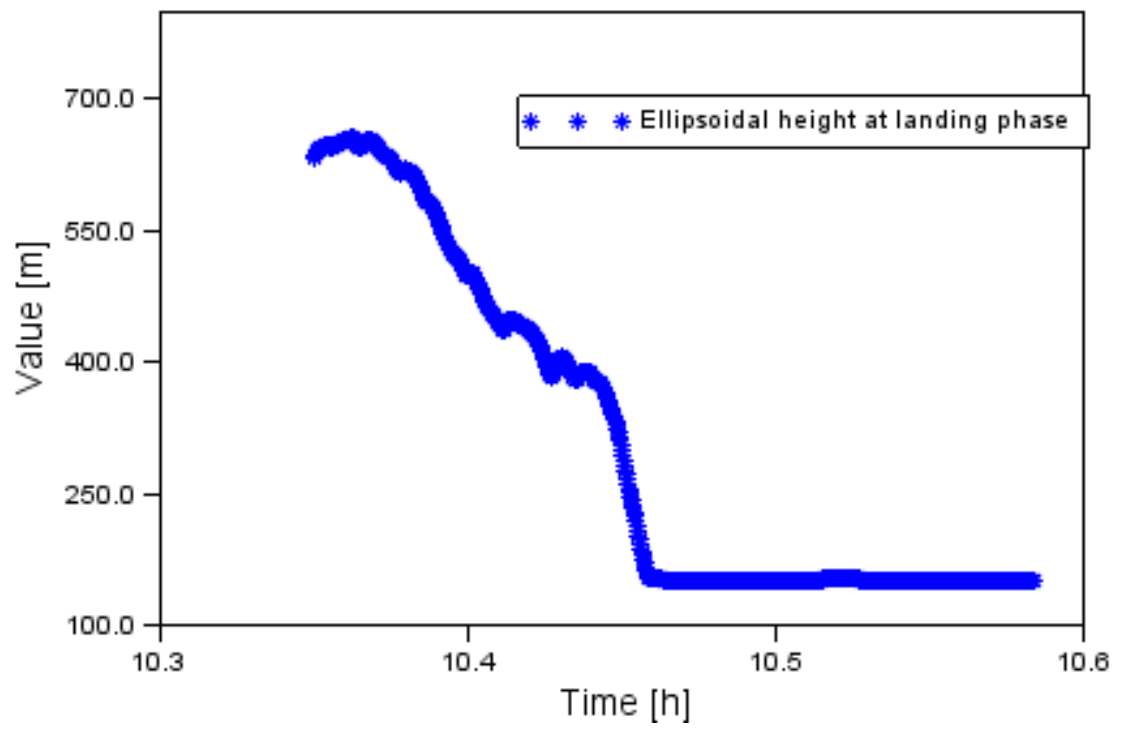

Fig. 2. Ellipsoidal height during the landing phase

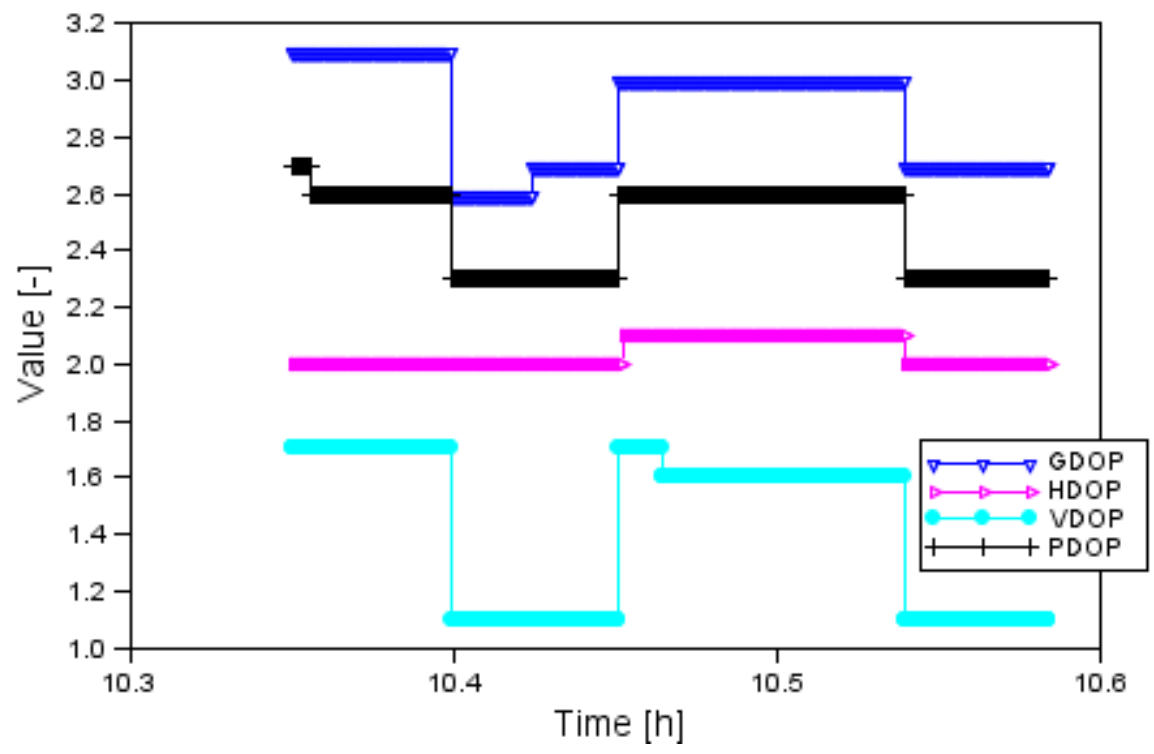

Fig. 3. Values of DOP coefficients

Figure 4 shows the accuracy of geocentric coordinates $X Y Z$ for the Cessna 172 aircraft based on the solution obtained in the GAPS programme. The average accuracy of determining the $X$-coordinate of the aircraft equals $4.1 \mathrm{~cm}$, with the discrepancy of the findings ranging from $3.9 \mathrm{~cm}$ to $4.3 \mathrm{~cm}$. The median parameter for the standard deviation of the $X$-coordinate is equal to $4.2 \mathrm{~cm}$. The average accuracy of determining the $Y$-coordinate of the aircraft is $3.6 \mathrm{~cm}$, with the discrepancy of the results ranging from $3.5 \mathrm{~cm}$ to $3.8 \mathrm{~cm}$. The median parameter for the standard deviation of the $Y$-coordinate is equal to $3.6 \mathrm{~cm}$. The average accuracy of determining the $\mathrm{Z}$ coordinate of the aircraft is $4.8 \mathrm{~cm}$, with the discrepancy of the results ranging from $4.3 \mathrm{~cm}$ to $5.7 \mathrm{~cm}$. The median parameter for the standard deviation of the Z-coordinate is equal to $4.8 \mathrm{~cm}$. The results of the accuracy of $X Y Z$ coordinates of the aircraft Cessna 172 during the approach to landing are much higher than $10 \mathrm{~cm}$.

Figure 5 shows the MRSE (Mean Radial Spherical Error) parameter values for XYZ coordinates of the Cessna 172 aircraft based on the solution obtained in the GAPS programme. The MRSE parameter specifies the position error of the determined coordinates of the aircraft Cessna 172. Furthermore, the MRSE parameter is determined on the basis of the obtained from the accuracy of $X Y Z$ coordinates (see 
Figure 4) [12]. A typical value of the MRSE parameter is $7.3 \mathrm{~cm}$, with the dispersion of the results ranging from $7.1 \mathrm{~cm}$ and $8.0 \mathrm{~cm}$. The median value for the MRSE parameter is equal to $7.2 \mathrm{~cm}$.

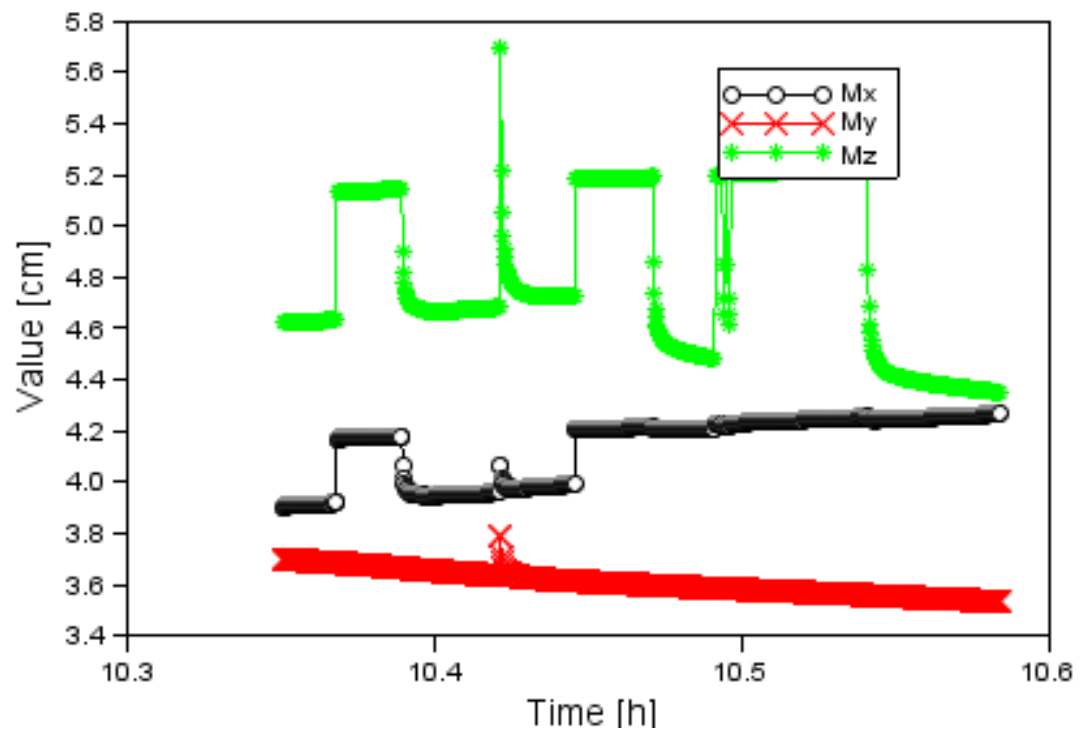

Fig. 4. Accuracy of positioning of Cessna 172 plane at phase of landing

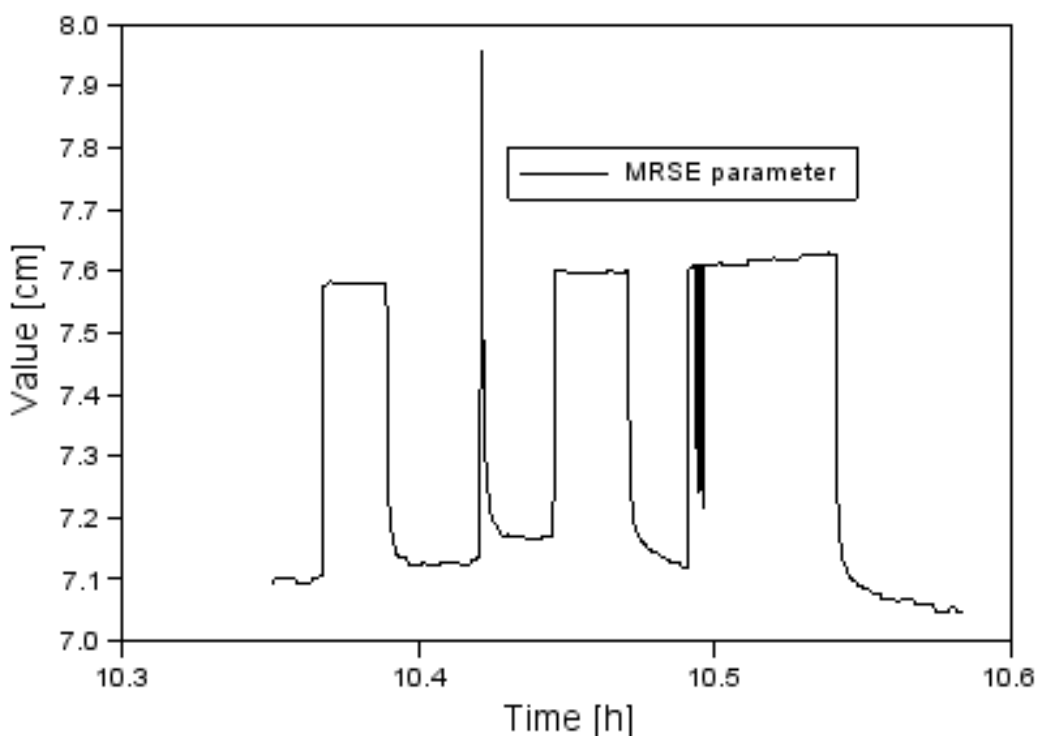

Fig. 5. Values of MRSE parameter

In Figure 6 we verified the determined $X Y Z$ coordinates of the aircraft in the GAPS programme. For this purpose, we determined the difference in the $X Y Z$ coordinates of the aircraft between the solution made in the GAPS programme and in the CSRS-PPP programme [13]. The average value of the difference in the $X$-coordinate is equal to $0.481 \mathrm{~m}$ and the RMS (Root Mean Square) bias of this difference equals $0.291 \mathrm{~m}$. The discrepancy of the results for the $X$-coordinate difference ranges from $-0.026 \mathrm{~m}$ to $1.110 \mathrm{~m}$. The average value of the $Y$-coordinate difference is equal to $-0.091 \mathrm{~m}$ and the RMS bias of this difference is $0.209 \mathrm{~m}$. In addition, the amplitude of the results obtained for the difference in the $Y$-coordinate varies between $-0.254 \mathrm{~m}$ and $0.366 \mathrm{~m}$. The average value of the $Z$-coordinate difference equals $0.082 \mathrm{~m}$, and the RMS bias of this difference is $0.117 \mathrm{~m}$. In addition, the amplitude of the results obtained for the difference in the $\mathrm{Z}$ coordinate is from $-0.499 \mathrm{~m}$ to $0.272 \mathrm{~m}$.

Figure 7 illustrates a comparison of the $X Y Z$ coordinates of the aircraft made in the GAPS programme and the numerical application magicPPP [14]. For this purpose, we determined the difference in the $X Y Z$ coordinates of the aircraft between the solution made in GAPS and magicPPP. The average value of the difference in the $X$-coordinate is equal to $0.229 \mathrm{~m}$ and the RMS bias of this difference equals $0.435 \mathrm{~m}$. The discrepancy of the results for the $X$-coordinate difference ranges from 
$-0.404 \mathrm{~m}$ to $1.499 \mathrm{~m}$. The average value of the $Y$-coordinate difference is equal to $-0.223 \mathrm{~m}$ and the RMS bias of this difference is $0.166 \mathrm{~m}$. In addition, the amplitude of the results obtained for the difference in the Y-coordinate varies between $-0.566 \mathrm{~m}$ and $0.325 \mathrm{~m}$. The average value of the $Z$-coordinate difference equals $0.659 \mathrm{~m}$, and the RMS bias of this difference is $0.594 \mathrm{~m}$. In addition, the amplitude of the results obtained for the difference in the $Z$-coordinate is from -0.839 to $1.727 \mathrm{~m}$.

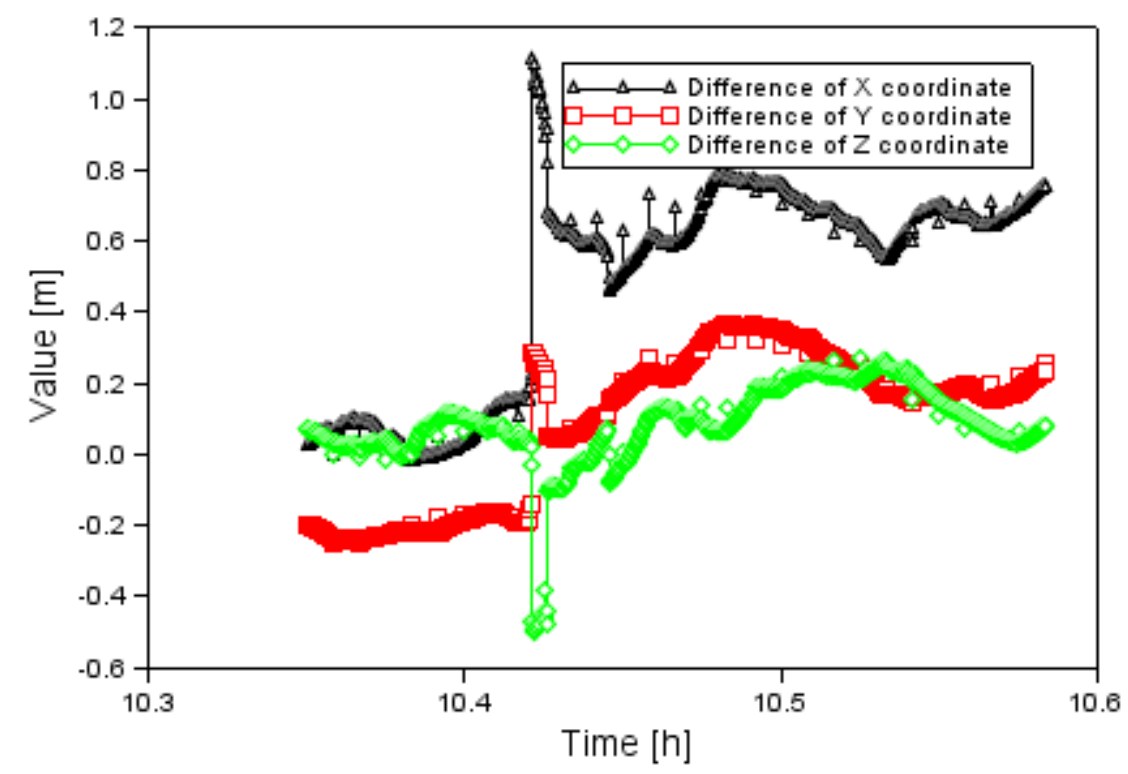

Fig. 6. Difference of $X Y Z$ coordinates of Cessna 172 plane based on GAPS and CSRS-PPP solutions

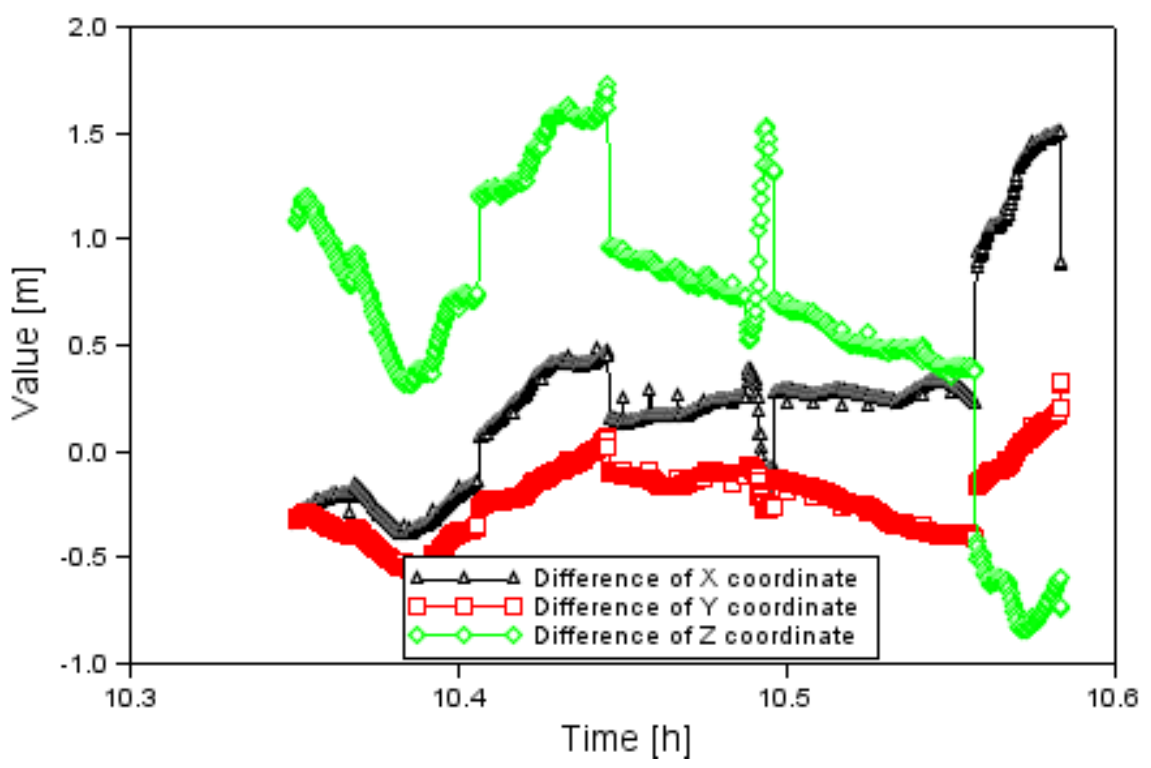

Fig. 7. Difference of $X Y Z$ coordinates of Cessna 172 plane based on GAPS and magicPPP solutions

The average value of the difference in the $X$-coordinate is smaller between the GAPS solutions and magicPPP compared to the solutions made in the GAPS and CSRS-PPP. On the other hand, the dispersion of the obtained results of the $X$-coordinate is smaller when it is made in GAPS and CSRSPPP rather than in the comparison between GAPS and magicPPP. The convergence of the value of the $Y$-coordinate of the GAPS is better with the results from the CSRS-PPP, than from magicPPP. In addition, the amplitude of comparing the difference in the $Y$-coordinate in the GAPS and CSRC-PPP programmes is less than between the solutions made in the GAPS and magicPPP programmes. The average value of the difference in the Z-coordinate is definitely smaller between the GAPS and CSRSPPP solutions rather than resulting from the comparison of solutions made in GAPS and CSRS-PPP. 
Moreover, the dispersion of findings of the Z-coordinate is smaller between the GAPS and CSRS-PPP solutions rather than in the case of the comparison of the solutions made in GAPS and magicPPP.

\section{Conclusions}

The PPP method is becoming more commonly used in GNSS satellite measurements as well as in aviation experiments. The article presents the possibility of using the PPP method in order to determine the $X Y Z$ geocentric coordinates of the aircraft for the augmentation system ABAS in air transport. The coordinates of the aircraft have been determined in the GAPS programme on the basis of the method of precise positioning PPP. In the computations we also used GPS code-phase observations from the Topcon HiperPro receiver to recover the position of the aircraft. The Topcon HiperPro receiver was fixed on board the Cessna 172 aircraft to accumulate and register GNSS coordinates. The work shows the following values: $X Y Z$ coordinates of the aircraft, DOP coefficients, the accuracy of the $X Y Z$ coordinates of the aircraft. In the article we also verified the determined coordinates of the Cessna 172 aircraft in the GAPS programme. For control reasons, we compared the $X Y Z$ aircraft coordinates computed in the GAPS, magicPPP and CSRS-PPP programmes. In the coming years, the PPP method may become a leading technique for precision positioning of aircrafts in air navigation. The PPP method will also play a significant role in air transport in the framework of the activities and the functioning of the augmentation system ABAS.

\section{References}

1. International Civil Aviation Organization. ICAO standards and recommended practices (SARPS), Annex 10 volume I (Radio navigation aids), 2006. [online] [01.01.2017]. Available at: http://www.ulc.gov.pl/pl/prawo/prawo-mi \%C4 \%99dzynarodowe/206-konwencje.

2. Krzykowska K., Siergiejczyk M.. Signal monitoring as a part of maintenance of navigation support system in civil aviation. Archives of Transport System Telematics, vol. 7, 2014, pp. 36-40.

3. Jafernik H., Krasuski K., Michta J. Assessment of suitability of radionavigation devices used in air. Scientific Journal of Silesian University of Technology. Series Transport. 2016, vol. 90, pp. 99-112.

4. Martín A., Anquela A., Berné J., Sanmartin M. Kinematic GNSS-PPP results from various software packages and raw data configurations. Scientific Research and Essays, Vol. 7(3), 2012, pp. 419-431.

5. Stępniak K., Wielgosz P., Paziewski J. Analysis of PPP accuracy depending on observing session duration and GNSS systems used. Biuletyn WAT, Vol. LXI., Nr 1, 2012, pp. 429-450 (in Polish).

6. Sanz Subirana J., Juan Zornoza J. M., Hernández-Pajares M. GNSS Data Processing, Volume I: Fundamentals and Algorithms. Publisher: ESA Communications, ESTEC, Noordwijk, Netherlands, ISBN 978-92-9221-886-7, 2013, pp. 151-154.

7. Leandro R., Santos M., Langley R. Analyzing GNSS data in precise point positioning software. GPS Solutions, vol. 15, Issue 1, 2011, pp. 1-13.

8. Hadaś T. GNSS-WARP software for Real-Time Precise Point Positioning. Artificial Satellites, Vol. 50, No. 2, 2015, pp. 59-76.

9. Ćwiklak J., Jafernik H. The monitoring system for aircraft and vehicles of public order services based on GNSS. Annual of Navigation, No. 16, 2010, pp. 15-24.

10. Website of GAPS service. [online] [01.01.2017]. Available at: http://gaps.gge.unb.ca/submitbasic.php.

11. Seeber G. Satellite Geodesy, 2nd completely revised and extended edition. Publisher: Walter de Gruyter GmbH \& Co. KG, 10785 Berlin, Germany, ISBN 3-11-017549-5, 2003, pp. 301-302.

12. Osada E. Geodesy. Oficyna Wydawnicza Politechniki Wrocławskiej, Wrocław, ISBN 837085-663-2, pp. 237-241 (in Polish).

13. Malinowski M., Kwiecień J. A comparative study of Precise Point Positioning (PPP) accuracy using online services. Reports on Geodesy and Geoinformatics, vol. 102, 2016, pp. 15-31.

14. Garcia A. M., Piriz R., Lainez Samper M. D., Romay Merino M. M. Multisystem Real Time Precise-Point-Positioning, today with GPS+GLONASS in the near future also with QZSS, Galileo, Compass, IRNSS. Paper presented at International Symposium on GPS/GNSS, Taiwan, 26-28 October 2010, pp. 1-6. 